Arq. Bras. Med. Vet. Zootec., v.68, n.6, p.1557-1562, 2016

\title{
Uso do Clamp and Rod Internal Fixation pedicular na estabilização da coluna lombossacra em felino: relato de caso
}

\author{
[The Clamp and Rod Internal Fixation pedicular stabilization of the lumbosacral spine in \\ feline: case report] \\ F. Paes, C.R.A. Ferrigno, P.V.T. Marinho, Í.S. Dal-Bó, J.F. Santos, D.T. Komorizono, \\ V.S. Galeazzi, T. Bregadioli
}

Universidade de São Paulo - São Paulo, SP

\begin{abstract}
RESUMO
Objetivou-se reportar, pela primeira vez descrita até então, a fixação de uma fratura de sétima vértebra lombar (L7) com luxação de L7-S1, por meio de fixação pedicular, utilizando-se Clamp and Rod Internal Fixation (CRIF) em um gato. Um felino de um ano de idade, macho, SRD, foi atendido com histórico de atropelamento havia sete dias, apresentando paraparesia não ambulatória secundária à fratura da sétima vértebra lombar e compressão da cauda equina. Após avaliação geral do paciente, no dia seguinte, ele foi submetido à cirurgia descompressiva por meio de laminectomia dorsal e posterior estabilização pedicular entre L7 e S1, utilizando-se CRIF bilateralmente. O paciente apresentou evolução favorável com deambulação normal após 15 dias do procedimento cirúrgico.
\end{abstract}

Palavras-chave: fratura vertebral, neurocirurgia, gatos

\begin{abstract}
The aim was to report the establishment of a seventh lumbar vertebra fracture (L7), the first described so far, with L7 -S1 luxation through pedicle fixation using Clamp and Rod Internal Fixation (CRIF) on a cat. A 1 year old male mongrel feline was attended with a history of being hit by car seven days prior, with no ambulatory paraparesis secondary to fracture of the seventh lumbar vertebrae and compression of the cauda equina. After general evaluation of the patient, the next day, the patient was submitted to decompressive surgery through dorsal laminectomy and posterior pedicle stabilization between L7 and S1 using CRIF bilaterally. The patient presented a favorable outcome with normal ambulation after 15 days of surgery.
\end{abstract}

Keywords: vertebral fracture, neurosurgery, cats

\section{INTRODUÇÃO}

Fraturas e luxações vertebrais (FLV) são a maior causa de injúrias neurológicas nos pequenos animais. Tais injúrias são mais comumente associadas a trauma severo, ocorrendo em aproximadamente $6 \%$ dos casos apresentados com déficits neurológicos indicativos de disfunções medulares, sendo atropelamentos por carro responsáveis por 41 a $63 \%$ desses casos. A coluna vertebral toracolombar (T3-L3) é a região mais afetada, seguida pela coluna lombar (L4L7). FLV da região lombar constituem aproximadamente 25 a $30 \%$ de todas as FLV, e a região lombar caudal transmite forças propulsoras dos membros pélvicos, que predominantemente causam tração e compressão das vértebras, refletindo nos tipos comuns de FLV nesse local (Jeffery, 2010).

O fator determinante entre optar por um tratamento conservativo ou cirúrgico está em avaliar a instabilidade, sendo mais recentemente avaliada por meio da teoria dos três compartimentos, aplicada inicialmente em pacientes humanos e hoje adaptada para os cães. A teoria divide o corpo vertebral em três

Recebido em 12 de novembro de 2015

Aceito em 12 de maio de 2016

E-mail: fpaesmv@gmail.com 
compartimentos em seu comprimento, ou seja, dorsal (lâmina, processo espinhoso e ligamentos associados), médio (ligamento longitudinal dorsal, anel dorsal e córtex dorsal do corpo vertebral) e ventral (ligamento longitudinal ventral, anel ventral e córtex ventral do corpo vertebral); se dois ou mais compartimentos estiverem comprometidos, há instabilidade (Jeffery, 2010).

O objetivo do tratamento cirúrgico é a redução do segmento vertebral, a descompressão da medula espinhal e a estabilização rígida do canal medular. Existe uma infinidade de meios de estabilização descritos, porém aqueles que resultam nos melhores resultados hoje são: pinos/ parafusos e polimetilmetacrilato, placas em corpo de vértebra, fixação esquelética externa, fixação com parafusos pediculares (Bruce et al., 2008).

Um dos questionamentos atuais hoje é identificar implantes com propriedades mecânicas adequadas e versatilidade nos diferentes tipos de ossos e fraturas (Zahn e Matis, 2004). Pensando em um implante que pudesse ser utilizado em cirurgia veterinária com máxima versatilidade para aplicações clínicas, foi desenvolvido o Clamp and Rod Internal Fixation (CRIF) (Synthes, Switzerland), um sistema para osteossíntese extracortical interna composto de grampos que deslizam e se dispõem na posição desejada ao longo de uma haste de aço inoxidável, em que os parafusos são inseridos fixando firmemente os grampos na haste e o sistema no osso (Zahn e Matis, 2004).

Segundo Figueiredo (2013), em um estudo biomecânico que avaliou a estabilização da coluna toracolombar de cães, o CRIF promove rigidez e estabilidade, sendo inferior apenas a estabelecida pela placa bloqueada.

$\mathrm{Na}$ literatura revisada, não foi encontrado nenhum estudo ou relato clínico do uso de CRIF na estabilização da coluna lombossacra em felinos, afecção frequente na rotina clínica, o que estimulou a publicação do referido relato.

\section{CASUÍSTICA}

Foi atendido um felino, um ano de idade, SRD, com histórico de paraparesia não ambulatória, aquesia e incontinência urinária após um atropelamento com sete dias de evolução. Ao exame físico, o animal apresentava parâmetros fisiológicos dentro dos limites normais de referência e repleção vesical. Ao exame neurológico, os membros torácicos encontravamse normais e, na avaliação dos membros pélvicos, constatou-se ausência de propriocepção, reflexo flexor ausente e patelar diminuído. Havia ausência de dor superficial em membros pélvicos e presença da sensibilidade dolorosa profunda. $\mathrm{O}$ reflexo cutâneo do tronco encontrava-se presente em toda extensão. O paciente foi, então, imobilizado sobre superfície rígida e encaminhado para exame radiográfico simples de toda a coluna vertebral, porém com foco maior na região lombossacra, local da neurolocalização da lesão. Observou-se, portanto, fratura do terço médio de corpo de vértebra da sétima vértebra lombar (L7), com luxação da sétima vértebra lombar e primeira sacral (S1), além de uma fratura incompleta em asa do ílio direito (Fig. 1).

Após os exames clínicos e radiográficos, foi observado que, segundo a teoria dos três compartimentos, havia comprometimento do médio e ventral e optou-se pela realização da cirurgia descompressiva e estabilização da região identificada. $\mathrm{O}$ paciente permaneceu com a imobilização até o dia seguinte, quando foi realizada a cirurgia.

O paciente foi pré-anestesiado com Acepran ${ }^{\circledR}$ (Acepromazina, Cristália, SP, Brasil) $(0,05 \mathrm{mg} / \mathrm{kg} / \mathrm{IM})$ e Dimorf® (Morfina, Cristália, $\mathrm{SP}$, Brasil) $(0,5 \mathrm{mg} / \mathrm{kg} / \mathrm{IM})$. Após 15 minutos, a indução anestésica foi realizada com Propovan ${ }^{\circledR}$ (Propofol, Cristália, SP, Brasil) $(5 \mathrm{mg} / \mathrm{kg})$, e a manutenção com Isoflorane ${ }^{\circledR}$ (Isofluorano, Cristália, SP, Brasil) e oxigênio em sistema semifechado. Foi realizada terapia profilática com Cellozina ${ }^{\circledR}$ (Cefazolina, Rio Química Indústria Farmacêutica Ltda., SP, Brasil) $(22 \mathrm{mg} / \mathrm{kg}) 30$ minutos antes do início do procedimento.

O paciente foi posicionado em decúbito dorsal com os membros pélvicos mantidos sem estresse sob posição neutra. Realizou-se antissepsia com álcool $70 \%$ e iodopovidine e posterior posicionamento dos panos de campo. O acesso foi realizado por meio de uma incisão mediana dorsal desde a sexta vértebra lombar até a última vértebra sacral. Foi realizada divulsão da 
musculatura epaxial de ambos os lados dos processos espinhosos até a identificação da lâmina dorsal e o foco da fratura. Após tentativas de redução da fratura, sem muito sucesso, procedeu-se à realização da laminectomia dorsal com o auxílio de goivas, fresa cirúrgica de alta rotação e pinças Kerrison, seguindo a técnica descrita por Sharp e Wheeler, 2005. A lâmina dorsal de L7 e S1 foi removida até completa visualização e descompressão das raízes nervosas. Após a laminectomia, procedeu-se à fixação das vértebras (L7 e sacrais) utilizando-se um CRIF duplo, bilateral, uma barra do lado esquerdo com três standard clamps, sendo dois end clamps nas extremidades e um standard ao centro e, por fim, uma barra do lado direito com apenas um end clamp ao final dela (Fig. 2).

Para a implantação do CRIF, a divulsão foi ampliada de modo que os parafusos fossem fixados com segurança. Duas barras de 2,0mm foram retorcidas e cortadas em comprimento adequado, sendo posteriormente posicionadas em cada lado das vértebras acometidas, especificamente sobre a região do pedículo vertebral. Do lado direito, os mini clamps foram posicionados sobre os pedículos de L7 e S3 e posteriormente fixados com parafusos corticais 2,0mm autorrosqueantes após perfuração com broca de $1,5 \mathrm{~mm}$ e medição monocortical do pedículo. $\mathrm{O}$ mesmo procedimento foi realizado para o outro lado esquerdo, entretanto, deste lado, um mini clamp adicional foi posicionado de modo que se fixasse na faceta articular em direção ao corpo vertebral de S1. Foi realizado exame radiográfico pós-operatório, que evidenciou estabilização vertebral, porém sem redução vertebral adequada (Fig. 3).

Após esse período, o paciente foi liberado com recomendações de repouso absoluto e foram prescritos cloridrato de tramadol (4mg/kg/TID/VO/sete dias), dipirona (25mg/kg/ $\mathrm{VO} / \mathrm{BID} /$ quatro dias).

O animal apresentou melhora gradativa e, após quatro dias da cirurgia, ainda não permanecia em estação, porém já se esforçava para se levantar, recuperou o controle da micção e voltou a defecar normalmente. Cerca de oito dias após a cirurgia, voltou a deambular com dificuldade e aos 15 dias de pós-operatório já deambulava normalmente. No $15^{\circ}$ dia de pós-operatório, ao exame neurológico, constatou-se retorno da função normal dos membros pélvicos. Após três meses, foi realizada nova reavaliação neurológica. $\mathrm{Na}$ ocasião, o paciente apresentouse normal e recebeu alta médica.

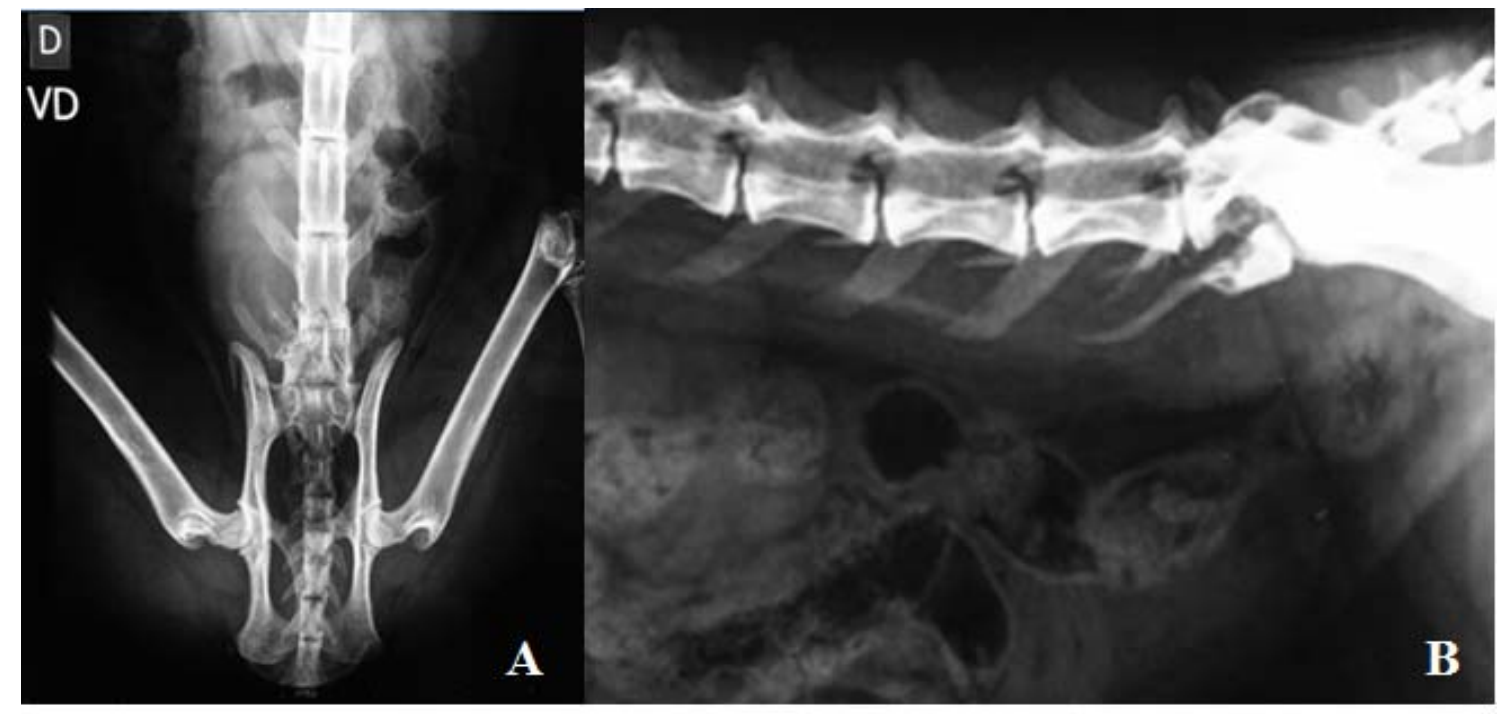

Figura 1. Fratura em corpo vertebral com luxação ventrodorsal da porção caudal de L7 visualizada em imagem radiográfica pré-operatória nas projeções ventrodorsal (A) e laterolateral (B). 


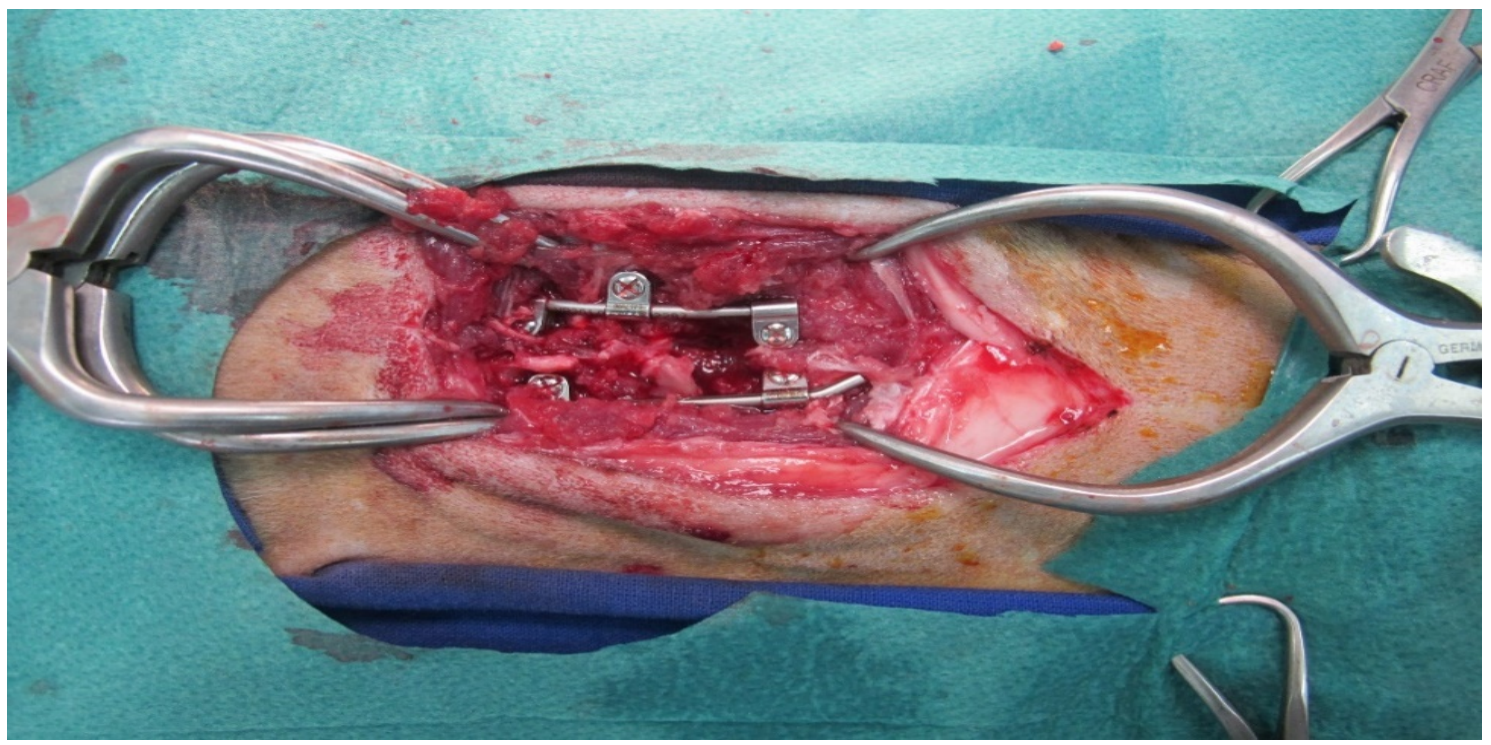

Fratura 2. Fixação pedicular utilizando Clamp and Rod Internal Fixation em fratura de L7 com luxação L7-S1.

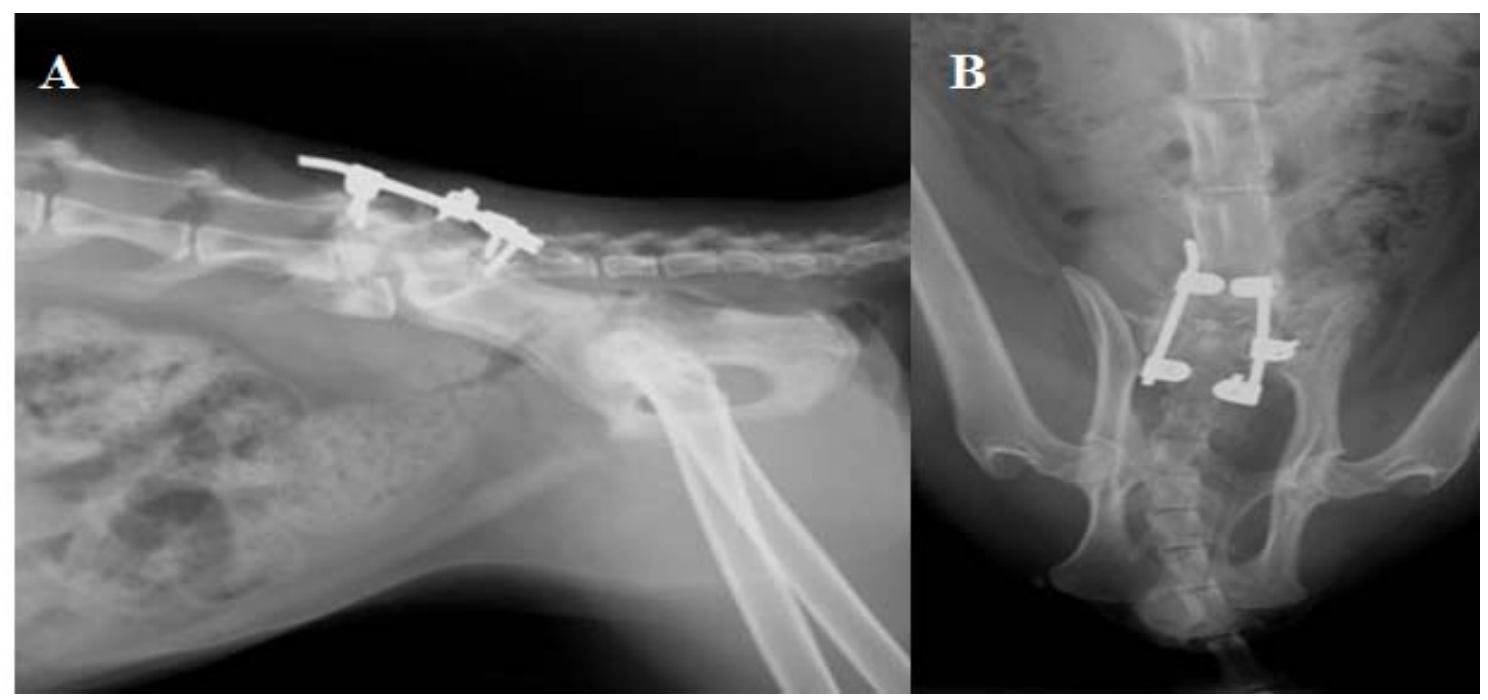

Figura 3. Imagem radiográfica de pós-operatório imediato na projeção laterolateral (A) e ventrodorsal (B) com duplo CRIF dorsolateral fixando a coluna lombossacra.

\section{DISCUSSÃO}

Fraturas no corpo vertebral de L7 são relativamente comuns $\mathrm{e}$ frequentemente diagnosticadas na rotina da clínica cirúrgica de pequenos animais, em geral como resultado de trauma automobilístico (Sharp e Wheeler, 2005).

A região lombossacra apresenta várias dificuldades na fixação, mais notavelmente no acesso lateral do corpo da vértebra. Deve-se perceber que o canal vertebral dessa região contém a cauda equina e que essa configuração carrega algumas implicações: recuperação após injúria espinhal ou de nervo periférico e perda da sensibilidade de dor profunda, que não carreia o mesmo prognóstico grave como seria para uma injúria em medula espinhal onde a compressão nervosa é muitas vezes extremamente dolorosa e requer descompressão rápida (Jeffery, 2010). 
Essas informações corroboram o caso relatado, em que, apesar do deslocamento inicial de mais de $100 \%$ do canal vertebral, não houve comprometimento neurológico grave, com manutenção da nocicepção.

Fraturas da sétima vértebra lombar afetam, principalmente, raízes nervosas correspondentes aos segmentos medulares de L6 até S1, as quais correspondem à inervação periférica dos nervos ciático e pudendo. Esses nervos são responsáveis pela condução dos reflexos flexor e perineal, respectivamente (Garibaldi et al., 2003). O paciente apresentou diminuição desses reflexos observada durante exame neurológico. Adicionalmente, constatou-se diminuição do reflexo patelar, o qual é conduzido pelo nervo femoral, que emerge dos segmentos medulares de L4 a L6. Esses segmentos localizam-se no nível das vértebras L3 e L4 do canal medular. Apesar da distância entre a fratura e esse segmento, essa alteração se justifica pelo edema e pela hemorragia que podem tem progredido em sentido cranial no canal medular. $\mathrm{O}$ nervo pudendo é responsável pela contração do esfíncter uretral externo e a incontinência observada é compatível com lesão desse nervo.

Independentemente da técnica utilizada, como regra geral, quanto maior a exatidão do alinhamento anatômico da coluna vertebral, melhor será o ambiente para a recuperação funcional da medula espinhal (Harrington e Bagley, 1998). O alinhamento de fraturas vertebrais em cães é auxiliado pelo conhecimento da anatomia vertebral normal, pela compreensão das forças que provocam deslocamentos vertebral e por instrumentos utilizados com cautela para manipulação vertebral. Resultados bem-sucedidos são provavelmente o resultado do alinhamento vertebral adequado com a descompressão do tecido nervoso resultante (Harrington e Bagley, 1998). Durante o processo de redução da fratura, é importante saber que a busca por uma redução perfeita é secundária ao objetivo de se evitar um agravamento ou uma lesão iatrogênica, uma vez que a medula tolera uma considerável deformação, ou seja, a redução incompleta sem mais trauma é preferível a uma redução mais adequada que, entretanto, causou prejuízo neural adicional (Jeffery, 2010). No caso relatado, em razão de o tempo de redução de fratura não ter sido ideal, houve dificuldade em realizar uma melhor redução anatômica da fratura, o que não impediu que o animal apresentasse uma boa evolução do quadro, principalmente quando associada ao uso de um implante que promove estabilização adequada, bem como ao conhecimento das características das estruturas neurológicas que compõem o canal vertebral nessa região, como elasticidade e resistência das fibras à tração, que, se levadas em consideração e fornecerem um ambiente adequado às raízes nervosas, podem então se recuperar (Jeffery, 2010). Em razão da contratura muscular, fibrose ou resposta inflamatória no local da fratura, evitou-se a manipulação excessiva em busca da redução anatômica mais adequada, pois, segundo Harrington e Bagley (1998), a manipulação excessiva pode agravar a lesão neurológica.

A descompressão geralmente é alcançada apenas com a estabilização das fraturas, sendo limitada a aplicação de laminectomia ou hemilaminectomia, salvo as situações em que se visualiza anel do disco intervertebral ou uma compressão do canal vertebral entre 35 a 50\% do diâmetro dele (Jeffery, 2010). No entanto, a laminectomia permite avaliar o interior do canal medular após a redução da fratura, possibilitando visibilizar possíveis fragmentos ósseos que possam causar danos às raízes nervosas ali presentes (Jeffery, 2010). No caso do referido estudo, por meio da laminectomia dorsal, foi possível remover pequenos fragmentos ósseos que comprimiam as raízes nervosas, bem como, após a estabilização, verificar uma melhor homogeneidade da disposição das raízes mesmo com o pequeno grau de redução alcançado.

O conhecimento das propriedades mecânicas de cada implante evita erros técnicos durante sua aplicação clínica. Embora não existam recomendações exatas para a rigidez ideal e a força necessária de um implante para controlar o movimento relativo de uma fratura durante $o$ processo de consolidação, um dispositivo de fixação com propriedades mecânicas semelhantes às do osso pode ser considerado uma fixação adequada (Zahn et al., 2008). O CRIF demonstrou, portanto, ser um dispositivo adequado para fraturas de L7 em gatos, uma vez que, além de ter o tamanho ideal para a espécie, apresenta facilidade no retorcimento, ajuste de comprimento, versatilidade no posicionamento dos parafusos em qualquer região da barra, bem como confere resistência adequada, já 
comprovada em estudos de ensaios biomecânicos (Figueiredo, 2013). Essas características observadas durante o procedimento cirúrgico no caso referido corroboram com Tomlinson (2005), o qual afirma que as principais propriedade do CRIF incluem excelente versatilidade, boa capacidade de contorno (retorcimento), facilidade de aplicação, mínima instrumentação e mínimo contato ósseo, além de ser financeiramente acessível e de força suficiente para permitir carga de peso imediata. No caso relatado, os parafusos foram aplicados exclusivamente nos pedículos vertebrais, no entanto poderia ter sido feita a aplicação dos parafusos envolvendo especificamente os pedículos e os corpos vertebrais de L7 e do sacro, o que possivelmente confere maior rigidez e resistência ao sistema, conforme relataram Meij et al. (2007), quando utilizaram parafusos pediculares na coluna lombossacra. Segundo Smolders et al. (2012), uma fixação lombossacra em corredores seguros deve ser realizada dorsocaudolateralmente para ventrocraniomedialmente, ou seja, proveniente da cortical dorsal da lâmina até a cortical dorsal do corpo vertebral, sem perfurar a porção cortical ventral assim como as paredes medial e lateral do pedículo. Além disso, sugere-se a perfuração de $20-50 \%$ de todo o comprimento do parafuso (Smolders et al., 2012).

Em conclusão, apesar de as fixações serem mais seguras quando envolvem o corpo vertebral pelo aporte ósseo, em razão de se tratar de um felino de porte pequeno, a fixação puramente pedicular com o CRIF mostrou-se eficiente, alcançando a recuperação neurológica esperada. Assim, este relato de caso apresenta mais uma lesão em que o CRIF demonstrou ser um implante com versatilidade e resistência necessárias para uma boa evolução clínica.

\section{REFERÊNCIAS}

BRUCE, C.W.; BRISSON, B.A.; GYSELINCK, K. Spinal fracture and luxation in dogs and cats: a retrospective evaluation of 95 cases. Vet. Comp. Orthop. Traumatol., v.21, p.280-284, 2008.
FIGUEIREDO, A.V. Estudo biomecânico ex vivo em coluna tóraco-lombar de cães com técnicas de estabilização utilizando placa bloqueada, clamp rod internal fixation, pino com cimento ósseo e técnica segmentar modificada 2013. 119f. Dissertação (Mestrado em Ciência) - Faculdade Medicina Veterinária e Zootecnia, Universidade de São Paulo, São Paulo, SP.

GARIBALDI, L.; PELLEGRINO, F.; SURANITI, A. Síndromes neurológicas em cães e gatos. São Caetano do Sul: Interbook, 2003. [328p.]

HARRINGTON, M.L.; BAGLEY, R.S. Realignment of a seventh lumbar vertebral fracture/luxation using a senn retractor in two puppies. J. Am. Anim. Hosp. Assoc., v.34, p.377-380, 1998.

JEFFERY, N.D. Vertebral fracture and luxation in small animals. Vet. Clin. N. Am. Samll Anim. Pract., v.40, p.809-828, 2010. Available in: http://dx.doi.org/10.1016/j.cvsm.2010.05.004.

Accessed in: 13 maio.2015.

MEIJ, B.P.; SUWANKONG, N.; VAN DER VEEN, A.J.; HAZEWINKEL, H.A.W. Biomechanical flexion-extension forces in normal canine lumbosacral cadaver specimens before and after dorsal laminectomy-discectomy and pedicle screw-rod fixation. Vet. Surg., v.36, p.742-751, 2007.

SHARP, N.J.H.; WHEELER, S.J. Small animal spinal disease: diagnosis and surgery. 2.ed. Edinburgh: Elsevier, 2005. 722p.

SMOLDERS, L.A.; VOORHOUT, G.; VAN DE VEN, R. et al. Pedicle screw-rod fixation of the canine lumbosacral junction. Vet. Surg., v.41, p.720-732, 2012.

TOMLINSON, J. Fractures of the distal femur. In: JOHNSON, A.L.; HOULTON, JOHN E.F.; VANNINI, RI. (Eds.). AO principles of fracture management in the dog and cat. Davos Platz: Thieme, 2005. p.297-303.

ZAHN, K.; FREI, R.; WUNDERLE, D. et al. Mechanical properties of 18 different AO bone plates and the clamp-rod internal fixation system tested on a gap model construct. Vet. Comp. Orthop. Traumatol., v.21, p.185-194, 2008.

ZAHN, K.; MATIS, U. The clamp rod internal fixator: application and results in 120 small animal fracture patients. Vet. Comp. Orthop. Traumatol., v.17, p.110120,2004 